\title{
Atención Sostenida y Selectiva en subtipos de TDAH y en Trastorno de Aprendizaje: una comparación clínica
}

\section{Silvia Álava Sordo, María Cantero-García ${ }^{2}$, Helena Garrido-Hernansaiz ${ }^{3}$, Iván Sánchez-Iglesias ${ }^{4}$, José Santacreu Más ${ }^{5}$}

${ }^{1}$ Departamento de Psicología Infantil. Centro de Psicología Álava Reyes.

2 Departamento de Psicología. Facultad de Ciencias Biomédicas y de la Salud. Universidad Europea de Madrid \& Área de Salud. Facultad de Psicología. Universidad Internacional de Valencia.

${ }^{3}$ Departamento de Educación y Psicología. Centro Universitario Cardenal Cisneros.

${ }^{4}$ Psychobiology and Behavioral Sciences Methods. Universidad Complutense de Madrid.

${ }^{5}$ Facultad de Psicología. Universidad Autónoma de Madrid

\section{España}

Correspondencia: María Cantero-García. Departamento de Psicología. Facultad de Ciencias Biomédicas y de la Salud. Universidad Europea de Madrid \& Área de Salud. Facultad de Psicología. Universidad Internacional de Valencia. Calle Pintor Sorolla, 21. 46002. Valencia. E-mail: maria.canterogar@gmail.com

(0) Universidad de Almería and Ilustre Colegio Oficial de la Psicología de Andalucía Oriental (Spain) 


\section{Resumen}

Introducción. El objetivo de este estudio es conocer si existen diferencias en atención selectiva y sostenida entre niños con diferentes subtipos de TDAH y niños con trastornos del aprendizaje (TA), para mejorar el diagnóstico diferencial entre TDAH y TA y entre los diferentes subtipos de TDAH.

Método. La muestra estuvo formada por 437 niños que acudieron a un centro de psicología, de los cuales 234 tenían TDAH y 203 TA (en los que se descartó un diagnóstico de TDAH). Se aplicaron pruebas de atención sostenida y selectiva (Caras, d2, EMAV, AGL, CSAT y CPT-II) a los participantes durante su evaluación y se realizaron análisis descriptivos, ANOVAs de un factor y pruebas F planeadas.

Resultados. Las diferencias entre los distintos indicadores de las pruebas utilizadas para medir la atención sugieren que los niños con TDAH tienen dificultades en el rendimiento en atención selectiva y sostenida y procesan la información de forma significativamente más lenta que los niños con TA.

Discusión y conclusiones. Los indicadores de algunas de las pruebas utilizadas podrían ser de utilidad para hacer un diagnóstico diferencial entre los niños con TDAH y con TA y para poder establecer un plan de intervención de tratamiento individualizado.

Palabras Clave: TDAH, atención sostenida, atención selectiva, trastorno de aprendizaje. 


\begin{abstract}
Introduction: The goal of this study is to examine whether there are differences in selective and sustained attention between children diagnosed with ADHD and children with LD, thus allowing to establish differences among these groups in several objective measures regarding attention and contributing to differential diagnosis between ADHD and LD.
\end{abstract}

Method The sample consisted of 437 children who attended a psychology center, of which 234 had ADHD and 203 had AD (in which a diagnosis of ADHD was ruled out). Sustained and selective attention tests (Caras, d2, EMAV, AGL, CSAT and CPT-II) were applied to the participants during their evaluation and descriptive analyzes, one-way ANOVAs and planned F tests were performed.

Results: The differences between the indicators of the tests used to measure attention suggested that children with ADHD have difficulties in performance in selective and sustained attention, and they process information significantly slower.

Discussion or Conclusion: The indicators of some of the tests used could be useful to make a differential diagnosis between children with ADHD and LD, and to design a personalized treatment intervention for each child.

Keywords: ADHD, sustained attention, selective attention, learning disorder. 


\section{Introducción}

Además de los síntomas de inatención, impulsividad e hiperactividad ya descritos en el Diagnostic and Statistical Manual of Mental Disorders 5 (APA, 2013), los niños con TDAH muestran déficits cognitivos como dificultades en la planificación, memoria de trabajo, inhibición y atención (Cortese et al., 2015) y tienden a tener más problemas con la atención focalizada o la velocidad de procesamiento de la información (análisis de los inputs y recuperación de la memoria almacenada; Barkley, 1990). Además, si bien se ha documentado que existe una alteración de la capacidad de atención sostenida en los niños con TDAH (Borger y Cassutto, 2014; Conners, 1994; de la Torre y de la Torre, 2003; González Castro, et al., 2014; Oades, 2000; Miranda et al. 2008), existe controversia en lo que respecta a la atención selectiva (Barkley, 1990; Crespo Eguillaz et al, 2006; de la Torre y de la Torre, 2003; Magaz et al., 2011; Rodríguez et al., 2009), algo que puede entenderse mejor atendiendo a los subtipos de TDAH existentes.

En este sentido, aunque el TDAH pueda considerarse una entidad única, sus manifestaciones pueden ser muy diversas, por lo que se pueden diferenciar varios patrones dentro de la misma alteración. Desde el DSM-IV (APA, 1994) se diferencian tres subtipos de TDAH que se han mantenido en el DSM-IV-TR (APA, 2000) y en DSM-5 (APA, 2013): predominante inatento, predominante hiperactivo impulsivo y combinado. La diferencia principal entre estos subtipos tiene que ver con el patrón de déficit atencional existente, pudiendo sugerir esto sustratos neurocognitivos diferentes para cada uno (Barkley, 1997).

A nivel general, los niños con subtipo inatento tienen más dificultades en la atención selectiva y sostenida, parecen ser más lentos a la hora de orientar la atención y responder a estímulos de su entorno y también tienen dificultades en la memoria verbal inmediata y diferida y en la flexibilidad cognitiva; sin embargo, no tienen dificultades para el control de la impulsividad (Arán y Mías, 2009; Solanto et al., 2007). Por otro lado, los niños con subtipo hiperactivo/impulsivo presentan dificultades en el control de impulsos, en la atención sostenida y en la memoria verbal inmediata y diferida (Arán y Mías, 2009; Barkley, 1990). Finalmente, en el subtipo combinado parece darse una elevada impulsividad, tanto cognitiva (vinculada a las tareas y al estilo general de aprendizaje) como motora (asociada a una falta de control motor; Balbuena, 2016; Belanger, Andrews, Gray y Korczak, 2018). 
En cuanto al proceso cognitivo de la atención, la mayoría de las investigaciones coinciden en que existe un déficit de la atención sostenida en todos los subtipos, aunque sobre todo en el inatento; sin embargo, en cuanto a la atención selectiva existe, como se indicaba anteriormente, una mayor controversia (Belanger et al., 2018). La literatura disponible al respecto sugiere que los déficits en la atención selectiva son más característicos del subtipo inatento y las alteraciones en la atención sostenida, del subtipo combinado (Balbuena, 2016; Jiménez, Rodríguez, Camacho, Alfonso y Artiles, 2015; Schmitz et al., 2002).

En resumen, cada subtipo de TDAH parece tener unas manifestaciones y características cognitivas diferentes donde la atención juega un papel importante, por lo que sería de gran importancia contar con instrumentos que permitan establecer un perfil neuropsicológico para cada uno de los subtipos (García-Savaté et al., 2012; Luo., Weibman, Halperin y Li, 2019). En este sentido, la precisión, el número de errores y, en menor medida, la velocidad de procesamiento son las tres variables claves en el uso de pruebas de evaluación a la hora de diferenciar diferentes perfiles (Santacreu, Shih y Quiroga, 2011). Así, los errores de omisión parecen estar más relacionados con los síntomas de inatención, mientras que los errores de comisión lo están con los síntomas de impulsividad (Epstein et al., 2003).

A este respecto, se han encontrado pocos estudios que evidencien un perfil diferente entre los niños con TDAH subtipo inatento y combinado y apenas hay estudios en el que se mencione el subtipo hiperactivo-impulsivo (por su escasa prevalencia), gozando los estudios existentes de problemas de validez interna y externa debido a que en muchos de ellos las muestras son muy reducidas (Reale, et al., 2017).

Por ejemplo, utilizando el Continuous Performance Test (CPT) de Conners, BaraJimenez, Vicuña, Pineda y Henao (2003) encontraron que en el subtipo combinado había una impulsividad significativamente mayor y una alteración en la capacidad de atención sostenida en comparación con el grupo inatento, en el cual solo se apreciaron dificultades en atención sostenida. Este resultado es parecido al obtenido en el estudio de Chiang y Gau (2008), el cual mostró que los niños con TDAH subtipo combinado eran más impulsivos (cometían más errores por comisión) y también tenían mayores dificultades en atención sostenida que el inatento. De forma similar, Fernández-Jaén et al. (2012) también encontraron usando el CPT mayores problemas de atención sostenida (mayor número de errores por omisión y mayor tiempo de reacción) en el subtipo combinado que en el inatento. Además, en el subtipo inatento hubo un 
mayor número de errores por comisión, lo que podría estar relacionado con una atención selectiva más deficitaria.

Siguiendo en la misma línea, cabe comentar el estudio de Arán y Mías (2009), quienes usaron varias pruebas para analizar el rendimiento atencional en los tres subtipos de TDAH. Los resultados indicaron que en el subtipo inatento hubo déficits tanto en la atención selectiva como en la sostenida, sin encontrarse alteraciones en el control de la impulsividad. En el subtipo hiperactivo/impulsivo, por su parte, hubo un rendimiento normal en atención selectiva y déficits notables tanto en atención sostenida como en el control de la impulsividad. Por último, en el subtipo combinado hubo más errores de comisión (sugiriendo mayor impulsividad) que en el subtipo inatento, resultado replicado en población china (Chiang y Gau, 2008) y EE. UU. (Hinshaw, Carte, Sami, Treuting y Zupan, 2002).

Sin embargo, otros estudios no han encontrado tales diferencias. También usando el CPT de Conners, Solanto et al., (2007) obtuvieron que tanto el subtipo inatento como el combinado tuvieron un mayor tiempo de reacción y un menor número de aciertos respecto al grupo control, sin mostrar diferencias entre los subtipos. De la misma forma, Chiang y Gau (2008) encontraron que el índice de discriminación $d$ ' fue menor en ambos subtipos de TDAH en comparación con un grupo control, sugiriendo una posible peor atención no solo sostenida sino también selectiva en ambos subtipos.

Además de las diferencias existentes entre los subtipos de TDAH, otra cuestión relevante para el diagnóstico de TDAH en niños y adolescentes es la elevada comorbilidad existente con otros trastornos, destacando especialmente los Trastornos del Aprendizaje (TA). Los TA se caracterizan por dificultades en la lectura, razonamiento, escritura o habilidades matemáticas, con un rendimiento en estas áreas por debajo de lo esperado para la edad cronológica, a pesar de que sensorialmente no se detecte ningún déficit (Johnson, 2017; Karande y Kulkarni, 2005). La prevalencia de TA en niños con TDAH se sitúa, de acuerdo con varios estudios, entre el 16\% y el 56\% (CADDRA, 2011; Efron et al., 2016; Lonergan et al., 2019; Oerbeck et al., 2017; Reale et al., 2017; Tistarelli, Fagnani, Troianiello, Stazi y Adriani, 2020). Sin embargo, tales datos no reflejan una prevalencia de comorbilidad real, sino dificultades para realizar adecuadamente el diagnóstico diferencial debido a la similitud de los síntomas (Peterson et al., 2017; Preston, Heaton, McCann, Watson y Selke, 2009). En este sentido, cabe señalar la escasez de estudios existentes donde se compare un grupo con TDAH con 
un grupo con TA, así como el pequeño tamaño de las muestras empleadas en los estudios existentes (e.g., de la Torre y de la Torre, 2003; Gónzalez-Castro et al., 2014; Miranda et al., 2008; Rodríguez et al., 2009).

Por ello, resulta necesario realizar estudios para esclarecer tanto las diferencias existentes en atención sostenida y selectiva en TDAH entre los subtipos inantento y combinado, como las diferencias entre estos subtipos y los TA. La aplicación de pruebas de atención podría contribuir así a conocer las características específicas de cada subtipo de TDAH y a realizar un diagnóstico diferencial entre ellos, así como realizar un diagnóstico diferencial entre TDAH y TA.

\section{Objetivos e hipótesis}

Los objetivos del estudio son dos: en primer lugar, comparar el rendimiento en las pruebas de atención continua CPT-II y CSAT y en las pruebas de atención selectiva de cancelación EMAV, d2, Caras-R y AGL en una muestra clínica compuesta por niños con TDAH y por niños con TA en los que se había descartado el diagnóstico de TDAH. En segundo lugar, comprobar si hay diferencias en la realización de las diferentes pruebas de atención, tanto sostenida como selectiva, entre los niños con TDAH inatento y combinado. Así, se establecen las siguientes hipótesis (ver descripción de los instrumentos mencionados más adelante).

Respecto a la comparación de TDAH con TA:

- Hipótesis 1: El grupo con TDAH obtendrá unas puntuaciones más bajas que el grupo con TA en los índices de atención sostenida ( $d^{\prime}$ discriminación del CSAT; $d^{\prime}$ y el índice de confianza del CPT-II) y cometerán un mayor número de errores (tanto de comisión como de omisión) en ambas pruebas.

- Hipótesis 2: El grupo con TDAH obtendrá unas puntuaciones más bajas que el grupo con TA en los índices de atención selectiva (CA y AS del EMAV; aciertos del Caras; aciertos del d2 y ET del AGL) y un mayor número de errores de omisión y comisión en las pruebas EMAV, Caras y d2.

- Hipótesis 3: El grupo con TDAH realizará la tarea de forma más lenta que el grupo con TA, por lo que tendrá unas puntuaciones más bajas en AS del EMAV; aciertos del Caras; TOT del d2, ET del AGL y tiempo del CSAT. 
Respecto a la comparación entre los subtipos de TDAH:

- Hipótesis 4: El grupo con TDAH subtipo combinado mostrará más impulsividad que el grupo inatento y el grupo con TA, por lo que el número de errores de comisión (del EMAV, Caras, d2, CSAT y CTP-II) y perseveraciones (del CPT-II) será mayor.

- Hipótesis 5: El grupo con TDAH subtipo inatento realizará la tarea de forma más lenta que el grupo con TDAH subtipo combinado y que el grupo con TA, por lo que tendrá unas puntuaciones más bajas en AS del EMAV; aciertos del Caras; TOT del d2, ET del AGL, y un mayor tiempo de tiempo de respuesta en el CSAT.

- Hipótesis 6: El grupo con TDAH subtipo inatento obtendrá unos peores resultados que el grupo combinado y el grupo con TA en los índices de atención selectiva (CA y AS del EMAV; aciertos del Caras; aciertos del d2 y ET del AGL) y tendrá un mayor número de errores de omisión y comisión en dichas pruebas (EMAV, Caras y d2).

- Hipótesis.7: El grupo con TDAH subtipo combinado obtendrá unos resultados peores que el grupo inatento y el grupo con TA en los índices de atención sostenida ( $d^{\prime}$ discriminación del CSAT; $d^{\prime}$ y confidence index del CPT-II) y cometerán un mayor número de errores en ambas pruebas.

\section{Método}

\section{Participantes}

Participaron 437 personas, 278 varones y 159 mujeres, que acudieron al centro de Psicología Álava Reyes por problemas de aprendizaje entre abril de 2003 y agosto de 2015. De los 437 participantes, 234 recibieron el diagnóstico de TDAH y 203 de TA en los que se descartó un diagnóstico de TDAH. Dentro del grupo de TDAH, 97 mostraron un perfil inatento (22.19\%) y $137(31.35 \%)$ combinado. La edad osciló entre 6 y 16 años $(M=10.30, D T=$ 3.09). Además, es necesario señalar que la muestra de este estudio se utilizará para otros manuscritos. Por otro lado, la mayoría de los participantes recibieron una intervención en el mencionado centro, la cual se realizó con posterioridad a las evaluaciones reportadas en este informe. 


\section{Instrumentos}

Pruebas de atención selectiva / búsqueda visual

- El test de percepción de diferencias «Caras» (Thurstone y Yela, 2009). Evalúa la aptitud para percibir rápida y correctamente semejanzas y diferencias entre los diferentes estímulos presentados. Consta de 60 elementos gráficos; cada uno de ellos formado por tres dibujos esquemáticos de caras con la boca, ojos, cejas y pelo representados con trazos elementales; dos de las caras son iguales y la tarea consiste en determinar cuál es la diferente y tacharla. La fiabilidad del test muestra un $\alpha$ de Cronbach de .91

- El test de atención d2 (Brickenkamp, 2004) evalúa varios aspectos de la atención selectiva y la concentración en niños a partir de 8 años, adolescentes y adultos. El test está formado por 14 líneas con 47 caracteres, 658 elementos en total. En el estudio de Brickenkamp (2004) se encuentra una buena consistencia interna, con $\alpha$ de Cronbach en la muestra de EEUU de entre .79 a .96 .

- La Escala Magallanes de Atención Visual EMAV (García-Pérez y Magaz, 2000). Consiste en la identificación de las figuras iguales al modelo entre un grupo de figuras diferentes. Para niños de 6 a 8 años de edad, consta de un total de 720 figuras, de las cuales 140 son iguales al modelo. En la EMAV-2, para niños de 9 a 18 años el tamaño de las figuras es más pequeño. Consta de 1820 figuras de las cuales 340 son iguales al modelo. La fiabilidad test-retest obtuvo unos coeficientes de correlación de Pearson ( $p$ $<.001)$ de .48 para la calidad de atención de la EMAV-1, .56 para la EMAV-2, .46 para la atención sostenida de la EMAV-1, y .60 para la EMAV-2.

- AGL, atención global-local (Blanca et al., 2005). Evalúa la rapidez y la precisión perceptivas en una tarea que exige la distribución de la atención en dos niveles de un estímulo visual. Se utilizan elementos gráficos, denominados estímulos jerárquicos, consistentes en figuras grandes, que representan el nivel global de patrón visual y cuyo contorno lo forman figuras más pequeñas, que representan el nivel local. El índice de fiabilidad test-retest muestra un coeficiente de fiabilidad de .80 en todos los índices. 


\section{Pruebas de atención sostenida}

- El CSAT (Children Sustained Attention Task, (Servera y Llabrés, 2004). Es una versión de las tareas de ejecución continua o CPTs, para medir la capacidad de atención sostenida de la infancia. El target o estímulo objetivo es doble (presencia de un 3 precedido de un 6,6-3). La respuesta es apretar la barra espaciadora del ordenador cada vez que se presente el target. Se presentan 600 estímulos en la pantalla durante 250 milisegundos. Los coeficientes de correlación entre bloques fluctúan alrededor de .80 para los aciertos, .85 para los tiempos de reacción y .80 para las comisiones.

- CPT de Conner II (2004). La prueba está compuesta por 6 bloques, con 3 sub-bloques que cada uno contiene 20 presentaciones de letras. La duración total de la prueba es de 14 minutos, y se puede aplicar a partir de los 6 años. Los datos de fiabilidad de dos mitades muestran unos coeficientes de correlación entre bloques de .83 para las comisiones, .94 para las omisiones y .95 para el tiempo de reacción.

\section{Análisis de datos}

Las puntuaciones en las diferentes pruebas de atención se describieron mediante su media y desviación típica. Se realizaron comparaciones de medias en las diferentes pruebas de atención entre los grupos (TDAH inatento, TDAH combinado y TA) mediante ANOVAs de un factor con comparaciones post hoc (con corrección de Boferroni para evitar el aumento de tasa de error tipo I por familia de comparaciones). Para comparar el grupo con TA y los dos grupos de TDAH tomados en conjunto (inatento y combinado) se utilizó una prueba $F$ planeada a priori. A pesar de que la distribución de las puntuaciones en diferentes pruebas de atención no se ajustaba a la distribución normal según el estadístico de Kolmogorov-Smirnov, el estadístico $F$ es robusto frente al incumplimiento del supuesto de normalidad (Pardo, Ruiz, y San Martín, 2010). Los análisis de datos se realizaron usando SPSS 20.

\section{Resultados}

Las comparaciones de medias de puntuaciones de atención entre los diferentes grupos de muestras clínicas son presentadas a continuación. Desde la Tabla 1 a la Tabla 7 se muestran estadísticos descriptivos de estas puntuaciones, y las comparaciones de medias y junto con los tamaños del efecto de las diferencias. Los resultados fueron agrupados según el tipo 
de atención que estábamos evaluando, la velocidad de ejecución y el control de la impulsividad.

Tabla 1. Descriptivos de Escalas de Atención Sostenida

\begin{tabular}{lcccccccccc}
\hline & \multicolumn{2}{c}{ TA (1) } & \multicolumn{3}{c}{$\begin{array}{c}\text { TDAH } \\
\text { inatento (2) }\end{array}$} & \multicolumn{3}{c}{$\begin{array}{c}\text { TDAH } \\
\text { combinado (3) }\end{array}$} \\
\hline Escala & $n$ & $M$ & $D T$ & $n$ & $M$ & $D T$ & $n$ & $M$ & $D T$ \\
Percentil $d^{\prime}$ CPT-II & 121 & 54.44 & 27.24 & 70 & 64.89 & 22.73 & 77 & 61.70 & 26.31 \\
Índice de confianza CPT-II & 121 & 52.58 & 20.20 & 69 & 65.68 & 22.71 & 76 & 72.20 & 23.75 \\
Percentil $d^{\prime}$ CSAT & 42 & 0.88 & 1.10 & 38 & 0.41 & 1.05 & 58 & 0.45 & 1.59 \\
Omisiones CPT-II & 120 & 52.75 & 13.20 & 68 & 63.65 & 18.52 & 72 & 68.46 & 24.13 \\
Comisiones CPT-II & 120 & 50.07 & 10.60 & 68 & 53.88 & 9.02 & 73 & 50.06 & 8.99 \\
Comisiones CSAT & 42 & 54.69 & 69.64 & 39 & 166.21 & 402.21 & 58 & 84.50 & 109.11 \\
\hline
\end{tabular}

Tabla 2. Comparación de Medias en las Escalas de Atención Sostenida

\begin{tabular}{lcccccccc}
\hline & \multicolumn{4}{c}{ ANOVA } & \multicolumn{4}{c}{$\begin{array}{c}\text { Comparación de medias } \\
\text { entre grupos }(p)\end{array}$} \\
\hline Escala & $F$ & $g l$ & $p$ & $\eta^{2}$ & $1-2$ & $1-3$ & $2-3$ & $1-(2$ y 3$)$ \\
Percentil $d^{\prime}$ CPT-II & 4.11 & 2,265 & $\mathbf{. 0 1 8}$ & .030 & $\mathbf{. 0 1 4}$ & .151 & .711 & $\mathbf{. 0 0 6}$ \\
Índice de confianza CPT-II & 20.33 & 2,263 & $<.001$ & .134 & $<.001$ & $<.001$ & .214 & $<.001$ \\
Percentil $d^{\prime}$ CSAT & 1.69 & 2,135 & .188 & .024 & .246 & .247 & .985 & .068 \\
Omisiones CPT-II & 18.69 & 2,257 & $<.001$ & .127 & $<.001$ & $<.001$ & .382 & $<.001$ \\
Comisiones CPT-II & 3.83 & 2,258 & $\mathbf{. 0 2 3}$ & .029 & $\mathbf{. 0 2 9}$ & 1.000 & .055 & .118 \\
Comisiones CSAT & 2.62 & 2,136 & .076 & .037 & .215 & .224 & .438 & $\mathbf{. 0 4 7}$ \\
\hline
\end{tabular}

Nota: 1 : TA; 2: TDAH inatento; 3 : TDAH combinado; (2 y 3): Grupos TDAH inatento y combinado tomados en conjunto. En negrita, valores $p$ significativos.

En la Tabla 1 se muestran las medias y desviaciones típicas de las puntuaciones percentil de los participantes, en las escalas de atención sostenida. En $d^{\prime}$ del CTP-II se encontraron diferencias significativas (Tabla 2) entre los participantes con TA y el grupo de TDAH inatento, y entre TA y ambos grupos de TDAH tomados en conjunto (observándose una mayor media, es decir un mayor número de dificultades, en los grupos de TDAH) con un tamaño del efecto $\eta^{2}=.030$. También hubo diferencias significativas en el índice de confianza y las omisiones CTP-II entre el grupo con TA y el TDAH inatento, el grupo con TA y los combinados y entre el entre TA y ambos grupos de TDAH tomados en conjunto con un tamaño del efecto $\eta^{2}=.134$ y $\eta^{2}=.127$ respectivamente. Los sujetos con TDAH tomándose en conjunto 
cometieron de forma significativa más comisiones en el CSAT que los TA con un tamaño del efecto $\eta^{2}=.037$.

Tabla 3. Descriptivos de Escalas de Atención Selectiva

\begin{tabular}{lcccccccccc}
\hline & \multicolumn{3}{c}{ TA (1) } & \multicolumn{3}{c}{ TDAH } & \multicolumn{3}{c}{ TDAH } \\
& \multicolumn{3}{c}{ inatento (2) } & \multicolumn{3}{c}{ combinado (3) } \\
\hline Escala & $n$ & $M$ & $D T$ & $n$ & $M$ & $D T$ & $n$ & $M$ & $D T$ \\
Percentil CA EMAV & 203 & 33.96 & 24.83 & 97 & 24.07 & 22.46 & 137 & 30.40 & 25.29 \\
Percentil AS EMAV & 203 & 49.63 & 27.60 & 97 & 40.98 & 26.87 & 137 & 46.79 & 30.24 \\
Percentil aciertos Caras & 129 & 52.69 & 27.67 & 70 & 35.61 & 24.88 & 79 & 46.95 & 28.05 \\
Percentil TA d2 & 146 & 48.07 & 28.78 & 70 & 30.76 & 29.81 & 83 & 36.53 & 28.11 \\
Percentil ET (ejecución total) & 41 & 38.20 & 24.40 & 11 & 16.55 & 13.31 & 8 & 33.13 & 28.53 \\
AGL & 201 & 2.78 & 13.92 & 96 & 2.29 & 8.49 & 134 & 2.09 & 6.52 \\
Comisiones EMAV & 127 & 1.70 & 2.16 & 69 & 2.43 & 5.01 & 74 & 2.24 & 2.88 \\
Comisiones Caras & 146 & 7.04 & 7.82 & 66 & 11.03 & 10.12 & 77 & 11.42 & 11.06 \\
Comisiones d2 & 201 & 17.93 & 24.51 & 96 & 22.67 & 26.77 & 134 & 19.04 & 23.28 \\
Omisiones EMAV & 127 & 0.79 & 4.39 & 69 & 1.45 & 8.52 & 74 & 0.65 & 2.69 \\
Omisiones Caras & 146 & 13.86 & 24.02 & 66 & 16.73 & 18.41 & 77 & 17.48 & 21.53 \\
Omisiones d2 & & & & & & & & & &
\end{tabular}

Tabla 4. Comparación de Medias en las Escalas de Atención Selectiva

\begin{tabular}{|c|c|c|c|c|c|c|c|c|}
\hline \multirow[b]{2}{*}{ Escala } & \multicolumn{4}{|c|}{ ANOVA } & \multicolumn{4}{|c|}{$\begin{array}{c}\text { Comparación de medias } \\
\text { entre grupos }(p)\end{array}$} \\
\hline & $F$ & $g l$ & $p$ & $\eta^{2}$ & $1-2$ & $1-3$ & $2-3$ & $1-(2$ y 3$)$ \\
\hline Percentil AS EMAV & 3.07 & 2,434 & .048 & .014 & .036 & .635 & .270 & .036 \\
\hline Percentil CA EMAV & 5.37 & 2,434 & .005 & .024 & .003 & .387 & .126 & .005 \\
\hline Percentil aciertos Caras & 9.01 & 2,275 & $<.001$ & .061 & $<.001$ & .301 & .031 & .001 \\
\hline Percentil TA d2 & 9.79 & 2,296 & $<.001$ & .062 & $<.001$ & .011 & .434 & $<.001$ \\
\hline Percentil ET AGL & 3.71 & 2,57 & .031 & .115 & .023 & .842 & .288 & .046 \\
\hline Comisiones EMAV & 0.17 & 2,428 & .840 & .001 & .931 & .838 & .990 & .579 \\
\hline Comisiones Caras & 1.31 & 2,267 & .272 & .010 & .298 & .499 & .936 & .113 \\
\hline Comisiones d2 & 7.36 & 2,286 & .001 & .049 & .015 & .007 & .974 & $<.001$ \\
\hline Omisiones EMAV & 1.21 & 2,428 & .299 & .006 & .270 & .915 & .514 & .224 \\
\hline Omisiones Caras & 0.46 & 2,267 & .634 & .003 & .695 & .983 & .653 & .694 \\
\hline Omisiones d2 & 0.81 & 2,286 & .447 & .006 & .658 & .479 & .978 & .215 \\
\hline
\end{tabular}

Nota: 1: TA; 2: TDAH inatento; 3: TDAH combinado; (2 y 3): Grupos TDAH inatento y combinado tomados en conjunto. En negrita, valores $p$ significativos. 
La Tabla 3 muestra las medias y desviaciones típicas de las puntuaciones percentil de los participantes en las escalas de atención selectiva. En la Tabla 4 se muestran las comparaciones de medias en dichas escalas. En los percentiles de atención sostenida (AS) y Calidad de Atención (CA) del EMAV, el grupo con TDAH inatento obtiene unas puntuaciones significativamente más bajas que los TA; y ambos grupos de TDAH tomados en conjunto también obtienen puntuaciones significativamente que el grupo con TA con un tamaño del efecto $\eta^{2}=$ .036 y $\eta^{2}=.003$ respectivamente. En el percentil de aciertos del Caras se observan diferencias significativas entre el grupo con TDAH inatento y los TA, entre ambos grupos de TDAH tomados en conjunto con los TA a favor de los TA y entre los grupos TDAH inatento y combinado siendo la media más baja la del grupo inatento con un tamaño del efecto $\eta^{2}=.061$. En el percentil de total de aciertos del d2, los TA obtuvieron una puntuación significativamente mayor que el grupo inatento, que el combinado y que ambos grupos de TDAH tomados en conjunto con un tamaño del efecto $\eta^{2}=.062$. En el percentil de total de aciertos (ET) del AGL, el grupo con TA obtuvo una puntuación significativamente más alta que los TDAH inatentos y que ambos grupos tomados en conjunto con un tamaño del efecto $\eta^{2}=.115$. Respecto a los errores de comisión y omisión de las pruebas EMAV, Caras y d2 solo encontramos diferencias significativas en las comisiones del d2, entre el grupo con TA y los inatentos, el grupo con TA y los combinados y los TA y ambos grupos de TDAH tomados en conjunto (observándose una menor media en el grupo con TA) con un tamaño del efecto $\eta^{2}=.049$.

En la Tabla 5 se muestran las medias y desviaciones típicas de las puntuaciones percentil de los participantes, en las escalas de control de la impulsividad.

\section{Tabla 5. Descriptivos de Escalas de Control de Impulsividad}

\begin{tabular}{lcccccccccc}
\hline & \multicolumn{3}{c}{ TA (1) } & \multicolumn{4}{c}{ TDAH } & \multicolumn{3}{c}{ TDAH } \\
& \multicolumn{1}{c}{$n$} & $M$ & $D T$ & $n$ & $M$ & $D T$ & $n$ & $M$ & $D T$ \\
\hline Escala & 201 & 2.78 & 13.92 & 96 & 2.29 & 8.49 & 134 & 2.09 & 6.52 \\
Comisiones EMAV & 127 & 1.70 & 2.16 & 69 & 2.43 & 5.01 & 74 & 2.24 & 2.88 \\
Comisiones Caras & 146 & 7.04 & 7.82 & 66 & 11.03 & 10.12 & 77 & 11.42 & 11.06 \\
Comisiones d2 & 120 & 50.07 & 10.60 & 68 & 53.88 & 9.02 & 73 & 50.06 & 8.99 \\
Comisiones CPT-II & 42 & 54.69 & 69.64 & 39 & 166.21 & 402.21 & 58 & 84.50 & 109.11 \\
Comisiones CSAT & 120 & 54.13 & 14.59 & 68 & 74.35 & 37.74 & 72 & 63.25 & 20.95 \\
Perseveraciones & & & & & & & & &
\end{tabular}


En las comisiones del d2 se encontraron diferencias significativas entre los TA y los TDAH inatentos, los TA y los TDAH combinados y entre los TA y ambos grupos de TDAH tomados en conjunto (observándose una mayor media en los grupos de TDAH) con un tamaño del efecto $\eta^{2}=.049$. El grupo con TDAH inatento comete de forma significativa más omisiones en el CTP-II que el grupo con TA con un tamaño del efecto $\eta^{2}=.029$. En las perseveraciones del CTP-II se encontraron diferencias significativas entre los participantes con TA y el grupo de TDAH inatento, entre los TA y los combinados y entre TA y ambos grupos de TDAH tomados en conjunto (observándose una mayor media en los grupos de TDAH) con un tamaño del efecto $\eta^{2}=.105$.

La Tabla 6 muestra las medias y desviaciones típicas de las puntuaciones percentil de los participantes, en las escalas de velocidad de ejecución. En la Tabla 7 se muestran las comparaciones de medias en dichas escalas. Los sujetos con TDAH inatento realizaron de forma significativa más lenta que los TA el Caras, el d2 el EMAV y el AGL; los sujetos con TDAH combinado realizan de forma significativamente más lenta que los TA el d2. Los inatentos realizan de forma significativamente más lenta que los combinados el Caras y los sujetos de ambos grupos de TDAH tomados en conjunto son significativamente más lentos que los TA en el Caras, d2, EMAV y AGL, con unos tamaños del efecto $\eta^{2}$ de entre .014 y .115.

Tabla 6. Descriptivos de Escalas de Velocidad de Ejecución

\begin{tabular}{|c|c|c|c|c|c|c|c|c|c|}
\hline \multirow[b]{2}{*}{ Escala } & \multicolumn{3}{|c|}{$\mathrm{TA}(1)$} & \multicolumn{3}{|c|}{$\begin{array}{c}\text { TDAH } \\
\text { inatento (2) }\end{array}$} & \multicolumn{3}{|c|}{$\begin{array}{c}\text { TDAH } \\
\text { combinado (3) }\end{array}$} \\
\hline & $n$ & $M$ & $D T$ & $n$ & $M$ & $D T$ & $n$ & $M$ & $D T$ \\
\hline Percentil Tiempo CSAT & 42 & 62.86 & 24.35 & 39 & 57.67 & 23.67 & 58 & 56.90 & 23.11 \\
\hline Percentil aciertos Caras & 129 & 52.69 & 27.67 & 70 & 35.61 & 24.88 & 79 & 46.95 & 28.05 \\
\hline Percentil TOT d2 & 148 & 48.86 & 27.94 & 68 & 31.66 & 30.00 & 83 & 37.61 & 28.26 \\
\hline Percentil AS EMAV & 203 & 49.63 & 27.60 & 97 & 40.98 & 26.87 & 137 & 46.79 & 30.24 \\
\hline Percentil ET (ejecución total) AGL & 41 & 38.20 & 24.40 & 11 & 16.55 & 13.31 & 8 & 33.13 & 28.53 \\
\hline
\end{tabular}


Tabla 7. Comparación de Medias en las Escalas de Velocidad de Ejecución

\begin{tabular}{|c|c|c|c|c|c|c|c|c|}
\hline \multirow[b]{2}{*}{ Escala } & \multicolumn{4}{|c|}{ ANOVA } & \multicolumn{4}{|c|}{$\begin{array}{c}\text { Comparación de medias } \\
\text { entre grupos }(p)\end{array}$} \\
\hline & $F$ & $g l$ & $p$ & $\eta^{2}$ & $1-2$ & $1-3$ & $2-3$ & $1-(2$ y 3$)$ \\
\hline Percentil Tiempo CSAT & 0.85 & 2,136 & .430 & .012 & .586 & .429 & .986 & 207 \\
\hline Percentil aciertos Caras & 9.01 & 2,275 & $<.001$ & .061 & $<.001$ & .301 & .031 & .001 \\
\hline Percentil TOT d2 & 9.74 & 2,296 & $<.001$ & .062 & $<.001$ & .012 & .409 & $<.001$ \\
\hline Percentil AS EMAV & 3.07 & 2,434 & .048 & .014 & .036 & .635 & .270 & .036 \\
\hline Percentil ET AGL & 3.71 & 2,57 & .031 & .115 & .023 & .842 & .288 & .046 \\
\hline
\end{tabular}

Nota: 1: TA; 2: TDAH inatento; 3: TDAH combinado; (2 y 3): Grupos TDAH inatento y combinado tomados en conjunto. En negrita, valores $p$ significativos.

\section{Discusión y Conclusiones}

En la primera hipótesis se quiso comprobar si el grupo con TDAH obtendría unos resultados más bajos en las tareas que miden atención sostenida que el grupo de sujetos con TA, dada la evidencia encontrada de las dificultades de atención sostenida en los sujetos con TDAH (Berger y Cassuto, 2014, Conners, 1994, Epstein et al., 2003; González-Castro et al., 2014, Oades, 2000; O’Dougherty et al., 1984; Losier et al., 1996 y Miranda et al., 2008). Los resultados obtenidos apoyaron dicha hipótesis. Lo que podría indicar que, a igualdad de dificultades de aprendizaje, las del TDAH se deben a la atención sostenida y los TA, no. Así, el grupo de sujetos con TDAH obtuvo unas puntuaciones significativamente más bajas en los índices del CTP-II (índice de sensibilidad d', índice de confianza, omisiones). Los resultados no fueron significativos en el índice de detectabilidad $d^{\prime}$ del CSAT, pero sí en el número de comisiones en dicha prueba. Estos resultados concuerdan con los encontrados por Soroa et al. (2009) con el CSAT. Los resultados son significativos en el CTP-II, Creemos que esto podría deberse a que la tarea del CPT-II es una tarea más sencilla, se trata de una prueba de cancelación de tarea, donde la instrucción es pulsar en todas las letras menos en la X, y la prueba CSAT es una tarea de vigilancia donde la orden es más difícil, se trata de pulsa ante el estímulo doble "cada vez que salga un 6 y a continuación un 3", por lo que los niños con TA pueden hacer peor dicha tarea sin por ello tener un déficit de atención.

En la Hipótesis 2 se anticipó que los sujetos con TDAH obtendrían unas puntuaciones menores que el grupo con TA en las pruebas que miden atención selectiva, tal y como se 
apuntaba en las investigaciones de Barkley (1990); Crespo Eguilaz et al. (2006); De la Torre y de la Torre (2003); Magaz et al. (2011); Santacreu et al. (2011) y Rodríguez et al. (2009) sobre las dificultades de atención selectiva en los TDAH. Los resultados obtenidos, de nuevo, apoyaron la hipótesis. Así, el grupo de sujetos con TDAH obtuvo unas puntuaciones significativamente más bajas que el grupo con TA en todos los índices globales de las pruebas de cancelación utilizadas: AS y CA del EMAV, Aciertos del Caras, Aciertos del d2 y ET del AGL. Los resultados de menores puntuaciones en el percentil de concentración y de aciertos del d 2 concuerdan con los obtenidos por Rodríguez et al. (2009) y un menor percentil en CA y AS del EMAV en el grupo de sujetos con TDAH en los del estudio de Magaz et al. (2011). Lo que podría implicar que las dificultades de aprendizaje de los niños con TDAH se deben a su baja a atención selectiva, lo que no ocurriría en los sujetos con TA.

Respecto a los errores, los resultados no fueron significativos en cuanto al número de errores de omisión en las pruebas Caras, d2 y EMAV ni en las comisiones del EMAV y del Caras, pero sí en las comisiones del d2. El grupo con TDAH cometió de forma significativa más errores de comisión en el d2 que el grupo con TA. Quizás esto pueda deberse a la dificultad de la tarea; siendo el d2 una tarea de mayor dificultad que el Caras y el EMAV (en el d2 hay que diferenciar entre d y p; mientras que en el Caras hay que elegir entre dibujos de caras diferentes, o en el EMAV entre dibujos de figuritas con la misma posición), de hecho, la media de errores del Caras para el grupo con TDAH fue muy baja. Autores como MorenoMéndez y Martínez-León (2010) plantean que el test Caras no tiene un adecuado poder discriminativo para el TDAH. Sin embargo, en nuestro estudio, aunque el número de errores en esta prueba no fue significativo, es decir, el grupo de sujetos con TDAH no cometió más errores que los sujetos con TA.

En la Hipótesis 3 planteábamos que los sujetos con TDAH realizarían de forma más lenta que el grupo con TA las pruebas de atención, dado que en estudios previos se ha evidenciado que los niños con TDAH procesan la información de forma más lenta (Barkley, 1990; Conners, 1994; González-Castro et al., 2014; Santacreu et al., 2011 y Soroa et al., 2009). En nuestra investigación encontramos que el grupo con TDAH realizó de forma significativamente más lenta el EMAV (obtuvo una menor puntuación en el índice AS, y un menor número de aciertos, que en este caso hace referencia a la cantidad de estímulos procesados en esta prueba) y obtuvo unas puntuaciones más bajas en el percentil de aciertos del Caras, en el percentil ET del AGL y en el percentil de número total de elementos procesados del d2; esto im- 
plicaría que los sujetos con TDAH han realizado de forma más lenta las tareas de EMAV, Caras, AGL y del d2 que el grupo con TA porque procesan una menor cantidad de estímulos. Sin embargo, los datos no fueron significativos para el tiempo del CSAT. Esto podría deberse a que, tanto en el Caras como en el EMAV, el AGL y en el d2 es el propio sujeto quien tiene que administrarse el tiempo. Sin embargo, en el estudio de Soroa et al. (2009), los resultados mostraban que los sujetos con TDAH obtenían un mayor tiempo de reacción en el CSAT.

A la vista de los resultados obtenidos, se apoya la hipótesis de que los TDAH procesan la información de forma más lenta en las pruebas de cancelación de lápiz y papel que los niños con TA. Para medir la velocidad con la que los sujetos procesan la información, hay autores que han utilizado el índice de Velocidad de Procesamiento del WISC-IV. Así, en los estudios realizados por Bustillo y Servera (2015); Fenollar-Cortés et al. (2015); Mayes y Calhoun (2006), San Miguel-Montes et al. (2010); Wechsler (2005); Yang et al. (2013) y Zhu y Chen (2013) se evidenciaron unas puntuaciones significativamente más bajas para el grupo de sujetos con TDAH en el índice de Velocidad de Procesamiento (VP) del WISC-IV, lo que implicaría un procesamiento más lento de la información. Fernández-Marcos (2017) concluye que es la velocidad con la que los sujetos realizan las pruebas el mejor predictor del TDAH, por lo que el tiempo predice mejor que los errores.

En la Hipótesis 4 se planteó que los sujetos con TDAH con predominio combinado realizarían las pruebas de forma más impulsiva que los sujetos con TDAH con predominio inatento y los sujetos con TA, tal y como postularon Arán y Mías (2009); Balbuena (2016); Bará-Jiménez et al. (2003); Barkley (1990); Chiang y Gau (2008); Eptstein et al. (2013) y Hinshaw et al. (2002); por lo que el número de errores por comisión en las pruebas administradas sería mayor. En este caso no se pudo confirmar dicha hipótesis dado que no hubo diferencias significativas entre el grupo de TDAH subtipo inatento con el subtipo combinado en ninguna de las variables relacionadas con impulsividad.

Sin embargo, la diferencia fue estadísticamente significativa entre el grupo de TDAH combinado y el de TA en el número de comisiones del d2 y en el número de perseveraciones del CPT-II. Es decir, los niños con TDAH combinado realizaron de forma significativamente más impulsiva las pruebas d2 y CPT-II que el grupo con TA, pero no que el grupo con TDAH subtipo inatento. Los niños con TDAH subtipo inatento cometieron de forma significativa más comisiones en el d2 y en el CPT-II que el grupo con TA, lo que podría estar relacionado 
con dificultades en la atención selectiva en este grupo, es decir tuvieron más dificultades a la hora de seleccionar los estímulos diana, lo que les lleva a cometer un número más elevado de errores (Arán y Mías, 2009; Balbuena, 2006; Chiang y Gau, 2008; Fernández-Jaén et al., 2012; Jiménez et al., 2015 y Schmitz et al., 2002).

Los resultados tampoco fueron estadísticamente significativos para el número de errores del Caras, del CSAT y del EMAV en ninguno de los grupos; pero tal y como hemos comentado anteriormente quizá pueda deberse a que la tarea del Test Caras es fácil y los errores no tienen un adecuado poder discriminativo para el TDAH, dado que tanto el grupo de sujetos con TA como los TDAH cometen un número muy bajo de errores de comisión en estas pruebas. (Moreno-Méndez y Martínez-León, 2010; Fernández-Jaén et al., 2012) tampoco encontraron diferencias significativas en las comisiones del d2. Nosotros encontramos diferencias estadísticamente significativas en el número de comisiones del d2 entre el grupo con TA y el subtipo inatento y entre el grupo con TA y el subtipo combinado, pero no entre ambos grupos entre sí. Los resultados no concuerdan con los obtenidos por Arán y Mías (2009); Chiang y Gau (2008) Hinshaw et al., (2002) y Epstein et al. (2003), que encontraron que el grupo con subtipo combinado cometía un mayor número de comisiones en el CPT-II. Sin embargo, en el estudio de Fernández-Jaén et al. (2012) era el grupo con subtipo inatento los que cometían un mayor número de comisiones en el CPT-II. En nuestro estudio, el grupo con subtipo inatento cometió más comisiones en el CPT-II que el grupo con TA, pero no que el grupo con TDAH combinado lo que podría significar que el TDAH subtipo inatento, tiene más dificultades que los niños con TA a la hora de discriminar el estímulo diana, lo que le hace cometer un número más elevado de errores tanto de comisión como de omisión.

En la hipótesis 5 suponíamos que el grupo con TDAH subtipo inatento realizaría las tareas presentadas de forma más lenta (Barkley, 1990 y Solanto et al., 2007) que el grupo con TDAH subtipo combinado y que el grupo con TA, por lo que obtendrían unas puntuaciones más bajas en CA y AS del EMAV; aciertos del Caras; total de respuestas del d2, (dado que procesarían una menor cantidad de estímulos), ET del AGL y en el tiempo del CSAT. En este caso solo encontramos una diferencia significativa entre los grupos de TDAH inatento vs combinado en el número de aciertos del Caras. Los sujetos con TDAH inatento realizaron la prueba Caras de forma más lenta que los niños con TDAH combinado y que los niños con TA. Además, la diferencia también fue significativa entre el grupo de niños con TDAH subtipo inatento y los niños con TA. 
En el caso del EMAV, el grupo de niños con subtipo inatento realizó la tarea de forma significativamente más lenta que los niños con TA, y procesaron un menor número de estímulos. Sin embargo, la diferencia no fue estadísticamente significativa entre el subtipo inatento frente al combinado. Si atendemos al número de aciertos del EMAV y al número total de elementos procesados en el d2, tanto el grupo con TDAH inatento como combinado obtuvieron un número significativamente menor que el grupo con TA, lo que puede deberse tanto a una menor rapidez como a un problema en la atención selectiva en ambos grupos (Arán y Mías, 2009; Balbuena, 2006; Chiang y Gau, 2008; García et al. 2012; Jiménez et al. 2015 y Schmitz et al. 2002). Las diferencias no fueron significativas en el tiempo del CSAT, pero tampoco lo eran entre el grupo de sujetos con TDAH y con TA, lo que podría deberse, tanto a las dificultades de atención selectiva del grupo con TDAH inatento respecto a los TA (seleccionan menos estímulos diana que el grupo con TA) como a una menor velocidad de ejecución de las tareas respecto al grupo con TA (realizan más despacio las pruebas de cancelación de lápiz y papel).

En la hipótesis 6 planteábamos que el TDAH con subtipo inatento obtendría unos peores resultados en los índices de atención selectiva que los sujetos con TDAH subtipo combinado y que el grupo con TA (Arán y Mías, 2009; Balbuena, 2006; Chiang y Gau, 2008; García et al. 2012; Jiménez et al. 2015 y Schmitz et al. 2002). En este caso se confirmó que el grupo con TDAH subtipo inatento obtuvo una menor atención selectiva que el grupo con TA, pero no que el grupo con TDAH subtipo combinado (dada que la única diferencia significativa entre grupos en el percentil de aciertos del Caras). En los índices globales del EMAV: CA y AS, encontramos que el grupo con TDAH subtipo inatento obtuvo una puntuación significativamente menor que los sujetos con TA, pero no que el grupo con subtipo combinado. Lo mismo ocurre en el número total de aciertos del d2; los TDAH subtipo inatento obtuvieron una menor puntuación que el grupo con TA, pero no que el grupo con subtipo combinado; y en el percentil de aciertos del Caras los niños con subtipo inatento obtuvieron unas puntuaciones significativamente más bajas que el grupo con TA y que el grupo con TDAH subtipo combinado. Respecto al número de errores no encontramos diferencias significativas ni el Caras, ni el EMAV; pero sí entre el grupo de TDAH subtipo inatento y el grupo con TA en el número de comisiones del d2. Un mayor número de comisiones en el grupo inatento estaría en consonancia con los resultados encontrados por Fernández-Jaén et al. (2012), lo que podría implicar que los niños con TDAH subtipo inatento tienen más dificultades en la atención se- 
lectiva que los TA, pero no que los combinados, porque los combinados también tienen dificultades en la atención selectiva.

En hipótesis 7 se planteó que el subtipo combinado obtendría unos resultados más bajos en los índices de atención sostenida (Arán y Mías, 2009, Balbuena, 2016; Bara-Jiménez et al., 2003; Barkley, 1990; Chiang y Gau, 2008; Eptstein et al., 2013; Fernández-Jaén et al., 2012; Jiménez et al., 2015; Schimitz et al., 2002) que el grupo de sujetos con TDAH subtipo combinado, y que el grupo con TA. En este caso no podemos confirmar dicha hipótesis. Los resultados no fueron significativos para ninguna variable entre el grupo con TDAH subtipo inatento y el combinado. Sin embargo, si lo fueron para el índice de confianza y en el número omisiones del CPT-II donde tanto el grupo de TDAH subtipo inatento, como el combinado obtuvieron una puntuación significativamente más baja que el grupo con TA, pero no hay una diferencia significativa entre los dos grupos de TDAH (inatento vs combinado). Estos resultados irían en consonancia con los datos aportados por los autores que indican que existe un déficit en la atención sostenida en ambos subtipos (Arán y Mías, 2009, Balbuena, 2016; BaraJiménez et al., 2003; Barkley, 1990; Chiang y Gau, 2008; Epstein et al., 2003; Fernández-Jaén et al., 2012; Jiménez et al., 2015). Como limitaciones a estudio es importante considerar que no se han utilizado todas las pruebas de atención existentes y que al igual que en los estudios anteriores, la muestra no es representativa de la población infantil de TDAH. Se trata de una muestra clínica de conveniencia. Los tamaños del efecto encontrados son pequeños, lo que podría deberse a que los grupos comparados se parece bastante, dado que todos tienen dificultades de aprendizaje.

En función de los datos encontrados, podemos concluir que:

1) De acuerdo con los estudios revisados, los sujetos con TDAH tuvieron dificultades en la atención sostenida. Lo que podría indicar que, a igualdad de dificultades de aprendizaje, las del TDAH se deben a la atención sostenida y los TA, no.

2) Los sujetos con TDAH tuvieron dificultades en la atención selectiva. En nuestra investigación, los sujetos con TDAH han obtenido puntuaciones significativamente más bajas en los índices globales de las pruebas de atención selectiva que los sujetos con TA. Lo que implicaría que las dificultades académicas de los TDAH podrían deberse a la atención selectiva y en los TA no. 
3) Los sujetos con TDAH procesaron la información de forma significativamente más lenta en las pruebas de cancelación (EMAV, d2, Caras y AGL) pero no en las pruebas de atención sostenida (CSAT). Lo que implicaría que los sujetos con TDAH son más lentos cuando ellos tienen que gestionar su propia ejecución, pero no cuando es una tarea ya programada en tiempos como ocurre en los CPTs.

4) Los sujetos con TDAH subtipo combinado no realizaron las pruebas de forma significativamente más impulsiva que el grupo con TDAH subtipo inatento. Así, no hubo ninguna diferencia significativa en el número de errores y perseveraciones entre ambos grupos (inatentos vs combinados) en ninguna de las pruebas administradas. Sin embargo, el grupo con TDAH subtipo combinado cometió un mayor número de perseveraciones en el CPT-II y comisiones que el grupo con TA, pero también ocurría lo mismo con el grupo de niños con subtipo inatento (cometían más perseveraciones y comisiones que los niños con TA en dicha prueba y en el d2). Esto también podría deberse a las dificultades ya comentadas en la atención selectiva y sostenida de los TDAH.

5) Los sujetos con TDAH subtipo inatento procesaron la información de forma significativamente más lenta que los niños con TDAH combinado y que los TA, en la prueba Caras, pero los resultados no fueron significativos para el resto de las pruebas administradas. No obstante, los niños con TDAH subtipo inatento realizaron todas las pruebas de cancelación (EMAV, d2, Caras y AGL) de forma significativamente más lenta que los niños con TA; mientras que los combinados sólo hicieron de forma significativamente más lenta que los niños con TA el d2. Es decir, los niños con TDAH subtipo inatento son más lentos procesando la información que los niños con TA, pero no que los combinados.

6) El grupo con TDAH subtipo inatento tuvo una menor atención selectiva que el grupo con dificultades de aprendizaje, pero no fue menor que en el grupo con TDAH subtipo combinado. Las puntuaciones fueron estadísticamente significativas entre el grupo de sujetos con TDAH subtipo inatento y el grupo con dificultades de aprendizaje en todas las pruebas, pero entre los combinados y los inatentos sólo lo fueron en el número de aciertos del Caras. Lo que podría deberse a que ambos grupos tienen dificultades en la atención selectiva. 
7) El grupo con TDAH subtipo combinado no tuvo una menor atención sostenida que el grupo con TDAH subtipo inatento, aunque sí que mostraron diferencias significativas en el índice de confidencia del CPT-II respecto al grupo de sujetos con TA. En el caso de los niños con TDAH subtipo inatento, estas diferencias fueron significativas con respecto al grupo con TA en todas las puntuaciones del CTP-II. Lo que podría implicar que ambos grupos tienen dificultades en la atención sostenida. En la clínica es importante conocer dónde están las dificultades de atención del niño tanto para establecer un correcto diagnóstico como para realizar una intervención lo más individualizada posible. De esta forma, la intervención se centraría en trabajar el aspecto de la atención donde el niño tuviese el problema.

\section{Referencias}

American Psychiatric Association (1994). Diagnostic and statistical manual of mental disorders (4th ed.). Washington, DC: Author.

American Psychiatric Association (2000). Diagnostic and statistical manual of mental disorders (4th ed.). Text revision. Washington, DC: American Psychiatric Association.

American Psychiatric Association (2013). Diagnosis and statistical manual of mental disorders (5th ed.). Arlington, VA: American Psychiatric Publishing.

Arán, V. y Mías, C. D. (2009). Neuropsicología del Trastorno por déficit de atención/hiperactividad: subtipos predominio déficit de atención y predominio hiperactivoimpulsivo. Revista Argentina de Neuropsicología, 13, 14-28.

Balbuena, F., (2016). La elevada prevalencia del TDAH: Posibles causas y repercusiones socioeducativas, Psicología educativa, 22, 81-85

Bará-Jiménez, S., Vicuña, P., Pineda, D. A., y Henao, G. C. (2003). Perfiles neuropsicológicos y conductuales de niños con trastorno por déficit de atención/hiperactividad de Cali, Colombia. Revista neurolología, 37(7), 608-615.

Barkley R. A. (2006). Comorbid disorders, social and family adjustment, and subtyping. In R. A. Barkley, Ed. Attention-deficit hyperactivity disorder: a handbook for diagnosis and treatment. 3rd ed. New York; London: The Guilford Press; p. 184-218.

Barkley, R. (2000). Niños hiperactivos. Como comprender y atender sus necesidades especiales ( $3^{\mathrm{a}}$ ed.) Barcelona: The Guilford Press.

Barkley, R. A. (1990). Attention-deficit hyperactivity disorder: A handbook for diagnosis and treatment. New York: Guilford Press. 
Barkley, R. A. (1994) Can neuropsychological tests help diagnose ADD/ADHD? The ADHD Report, 2, 1-3.

Barkley, R. A. (1997). Behavioral inhibition, sustained attention, and executive functions. Constructing a unifying theory of ADHD. Psychological Bulletin, 121, 65-94.

Bélanger, S. A., Andrews, D., Gray, C., y Korczak, D. (2018). ADHD in children and youth: Part 1-Etiology, diagnosis, and comorbidity. Paediatrics \& Child Health, 23(7), 447-453.

Berger, I., y Cassuto, H. (2014). The effect of environmental distractors incorporation into a CPT on sustained attention and ADHD diagnosis among adolescents. Journal of neuroscience methods, 222, 62-68.

Blanca, M. J, Zalabardo, C., Rando, B., López Montiel, D. y Luna, R. (2005). AGL Atención global-local. Madrid. TEA Ediciones.

Brickenkamp, R. (2004). Test de atención d2. Madrid: Tea ediciones.

Bustillo, M., y Servera, M. (2015). Análisis del patrón de rendimiento de una muestra de niños con TDAH en el WISC-IV. Revista de Psicología Clínica con Niños y Adolescentes, 2(2), 121-128.

CADDRA (2011). Canadian Attention Deficit Hyperactivity Disorder Resource Alliance. Canadian ADHD Practice Guidelines (3th Edition). Toronto: Autor.

Castillo, M.D. (2009). La atención. Madrid: Pirámide.

Chiang, M., y Gau, S. S. (2008). Validation of attention-deficit-hyperactivity disorder subtypes among Taiwanese children using neuropsychological functioning. Australian and New Zealand journal of psychiatry, 42(6), 526-535.

Conners, C. K. (1994) Conners' Continuos Performance Test Computer program 3.0 User's Manual. Toronto, ON: Multi-Health Systems.

Conners, C. K. (2004). Conners'CPT-II. Continuos Performance Test II for Windows. Toronto: MHS

Corkum, P. V., y Siegel, L. S., (1993) Is the Continuous Performance Task a valuable research tool for use with children with Attention Deficit-Hyperactivity Disorder? Journal Child Psychol Psychiatry, 34,1217-39.

Cortese, S., Ferrin, M., Brandeis, D., Buitelaar , J., Daley, D., Dittmann, R. W., . . y European ADHD Guidelines Group (EAGG) (2015). Cognitive training for attentiondeficit/hyperactivity disorder: Meta-analysis of clinical and neuropsychological outcomes from randomized controlled trials. Journal of the American Academy of Child and Adolescent Psychiatry, 54, $164-174$. 
Crespo-Eguílaz, N., Narbona, J., Peralta, F., y Repáraz, R. (2006). Medida de atención sostenida y del control de la impulsividad en niños: nueva modalidad de aplicación del Test de Percepción de Diferencias “Caras”. Infancia y Aprendizaje, 29(2), 219-232.

De la Torre Benítez, G. G., y de la Torre, A. G. (2003). Evaluación de la Atención mediante el Test de Cancelación Simple y Cancelación Condicionada [Batería Neuropsicológica Sevilla (BNS)] en niños con Transtorno por Déficit de Atención e Hiperactividad (TDAH). Revista española de neuropsicología, 5(2), 177-193.

Efron, D., Bryson, H., Lycett, K., y Sciberras, E. (2016). Children referred for evaluation for ADHD: comorbidity profiles and characteristics associated with a positive diagnosis. Child: Care, Health and Development, 42(5), 718-724.

Epstein, J. N., Erkanli, A., Conners, C. K., Kleric, J., Castello, J. E., y Angold, A., (2003), Relations between Continuous Performance Test performance measures and ADHD behaviors, Journal of Abnormal Child Psychology, 31(5), 543-554.

Fenollar-Cortés, J., Navarro-Soria, I., González-Gómez, C., y García-Sevilla, J. (2015). Detección de perfiles cognitivos mediante WISC-IV en niños diagnosticados de TDAH: ¿Existen diferencias entre subtipos?. Revista de Psicodidáctica, 20(1), 157-176.

Fernández-Jaén, A., Martín Fernández-Mayoralas, D., López-Arribas, S., Pardos-Véglia, A., Muñiz-Borrega, B., García-Savaté, C., Prados-Parra, B., Calleja-Pérez, B. MuñozJareño, N., y Fernández-Perrone, A. L. (2012). Habilidades sociales y de liderazgo en el trastorno por déficit de atención/ hiperactividad: relación con las capacidades cognitivo-atencionales. Actas España Psiquiatría; 40(3):136-46

Fernández-Marcos, T., de la Fuente, C., y Santacreu, J. (2017). Test-retest reliability and convergent validity of attention measures. Applied Neuropsychology: Adult, 1-9.

García, C., Prados, B., Calleja, B., Muñoz, N., y Fernández, A. L. (2012). Habilidades sociales y de liderazgo en el trastorno por déficit de atención/hiperactividad: relación con las capacidades cognitivo-atencionales. Actas España Psiquiatría, 40(3), 136-46.

García-Pérez; E. M., y Magaz, A. (2000) EMAV. Escalas Magallanes de Atención Visual. Manual de Referencia. Bizkaia: Grupo Albor-cohs.

Greenberg, L. M., Kindschi, C. L., Dupuy, T. R., y Hughes, S. J. (2007). T.O.V.A. Test Of Variables of Attention Continuous Perfomance Test. Manual. Los Alamitos, CA: The TOVA Company.

Greenberg, L.M. (1996). T.O.V.A.: Test of Variables of Attention. Clinical Guide. Los Alamitos: Universal Attention Disorder Inc. 
Jiménez, J. E., Rodríguez, C., Camacho, J., Afonso, M., y Artiles, C. (2015). Estimación de la prevalencia del trastorno por déficit de atención con o sin hiperactividad (TDAH) en población escolar de la Comunidad Autónoma de Canarias. European Journal of Education and Psychology, 5(1).

Johnson, B. (2017). Learning Disabilities in Children, Epidemiology, Risk Factors and Importance of Early Intervention. BMH Medical Journal, 4(1).

Karande, S. y Kulkarni, M. (2005). Specific learning disability, the invisible handicap. Indian Pediatrics, 42(4), 315-9.

Lehman, E. B.; Naglieri, J. A., y Aquilino, S. A. (2010). A national study on the development of visual attention using the cognitive assessment system. Journal of Attention Disorders, $14,15-24$.

Lonergan, A., Doyle, C., Cassidy, C., Mahon, S. M., Roche, R. A. P, Boran L., y Bramham, J. (2019). A meta-analysis of executive functioning in dyslexia with consideration of the impact of comorbid ADHD. Journal of Cognitive Psychology, 31(7), 725-749.

Losier, B. J. Mc Grath, P. J., y Klein, R. M. (1996). Error patterns on the continous performance test in non-medicated and medicated samples of children with and without ADHD: A meta-analytic review. Journal of Child Psychology and Psychiatry, 37, 971-987.

Luo, Y., Weibman, D., Halperin, J. M., y Li, X. (2019). A review of heterogeneity in attention deficit/hyperactivity disorder (ADHD). Frontiers in human neuroscience, 13, 42.

Magaz, A. et al., (2011). EMAV. Escalas Magallanes de Atención Visual. Manual de Referencia Actualizado. Bizkaia: Grupo Albor-cohs.

Mahone, E.M., Hagelthorn, K.M., Cutting, L.E., Schuerholz, L.J., Pelletier, S.F., Rawlins, C., et al. (2002). Effects of IQ on executive function measures in children with ADHD. Child Neuropsychology, 8, 52-65

Mayes, S. D. y Calhoun, S. L. (2006). WISC-IV and WISC-III profiles in children with ADHD. Journal of Attention Disorders, 9(3), 486-493.

Miranda, M. C., Barbosa, T., Muszkalt, M., Rodrigues, C., Sinnes, E., Coelho, ... y Bueno, O. (2008). Patterns of performance on the Conner's CPT in Children with ADHD and learning disabilities. Journal of Attention Disorders, 11(5), 588-598.

Moreno-Méndez, J. H., y Martínez-León, N. C. (2010). Conductas externalizantes, rendimiento académico y atención selectiva en niños con y sin hiperactividad. Psychologia: avances de la disciplina, 1(1), 39-53. 
Nigg, J. T. (2000). On inhibition/disinhibition in developmental psycho- pathology: Views from cognitive and personality psychology and work- ing inhibition taxonomy. Psychological Bulletin, 126, 220-246.

O’Dougherty, M. Neuchterlein, K. H. y Drew, B. (1984) Hyperactive and hypoxic children: Signal detection, sustained attention, and behaviour. Journal of Abnormal Psychology, 93, 178-191.

Oerbeck, B., Overgaard, K. R., Aspenes, S. T., Pripp, A. H., Mordre, M., Aase, H., Reichborn-Kjennerud, T., y Zeiner, P. (2017). ADHD, comorbid disorders and psychosocial functioning: How representative is a child cohort study? Findings from a national patient registry. BMC Psychiatry, 17, 23.

Oades, R. D. (2000). Differential measures of "sustained attention" in children with attentiondeficit/hyperactivity or tic disorders: relations to monoamine metabolism. Psychiatry Research, 93, 165-178.

Peterson, R. L., Boada, R., McGrath, L. M., Willcutt, E. G., Olson, R. K., y Pennington, B. F. (2017). Cognitive prediction of reading, math, and attention: Shared and unique influences. Journal of Learning Disabilities, 50(4), 408-421.

Park, M. H., Kweon, Y. S., Lee, S. J., Park, E. J., Lee, C., y Lee, C. U. (2011). Differences in performance of ADHD children on a visual and auditory continuous performance test according to IQ. Psychiatry investigation, 8(3), 227-233.

Reale, L., Bartoli, B., Cartabia, M., Zanetti, M., Costantino, M. A., Canevini, M. P., . . . Lombardy ADHD Group. (2017). Comorbidity prevalence and treatment outcome in children and adolescents with ADHD. European Child \& Adolescent Psychiatry, 26(12), 1443-1457.

Rodríguez, C., Álvarez, D., González-Castro, P., García, J., Álvarez, L., Núñez, J. C., González, J. A., y Bernardo, A. (2009), TDAH y TA en escritura: comorbilidad en base a la Atención y Memoria Operativa. European Journal of Education and Psychology, 2(3), 181-198.

Russell, E.W. (2001). Toward an explanation of Dodrill's observation: high neuropsychological test performance does not accompany high IQs. Clinical Neuropsycho, 15, 423428.

San Miguel Montes, L. E., Allen, D. N., Puente, A. E., y Neblina, C. (2010). Validity of the Wisc-IV spanish for a clinically referred sample of Hispanic children. Psychological Assessment, 22(2), 465-469. 
Santacreu, J., Shih Ma, P., y Quiroga, M. A. (2011) DiViSa Test de Discriminiación Visual Simple de Árboles. Manual. Madrid: TEA ediciones.

Schmitz, M., Cadore, L., Paczko, M., Kipper, L., Chaves, M., Rohde, L. A., ... y Knijnik, M. (2002). Neuropsychological performance in DSM-IV ADHD subtypes: An exploratory study with untreated adolescents. The Canadian Journal of Psychiatry, 47(9), 863869.

Servera, M., y Llabrés, J. (2015). CSAT-R Tarea de atención sostenida para niños revisada. Madrid: TEA Ediciones.

Servera, M., y Llabrés, J. (2004) CSAT Tarea de atención sostenida para niños. Madrid: TEA Ediciones.

Solanto, M. V., Gilbert, S. N., Raj, A., Zhu, J., Pope-Boyd, S., Stepak, B., ... y Newcorn, J. H. (2007). Neurocognitive functioning in $\mathrm{AD} / \mathrm{HD}$, predominantly inattentive and combined subtypes. Journal of abnormal child psychology, 35(5), 729-744.

Soroa, M., Iraola J.vA., Balluerka, N., y Soroa, G., (2009), Evaluación de la atención sostenida de niños con Trastorno por Déficit de Atención con Hiperactividad, Revista de Psicodidáctica, 14(1) 13-27

Thurstone, L. L., y Yela, M. (2009) Caras. Test de percepción de diferencias. Madrid. Tea ediciones.

Thurstone, L. L., y Yela, M. (2012) Caras-R. Test de percepción de diferencias. Madrid. Tea ediciones.

Tirapu, J., Ríos, M., y Maestú, F. (2008). Manual de neuropsicología. Barcelona: Viguera Editores.

Tistarelli, N., Fagnani, C., Troianiello, M., Stazi, M. A., y Adriani, W. (2020). The nature and nurture of ADHD and its comorbidities: A narrative review on twin studies. Neuroscience \& Biobehavioral Reviews, 109, 63-77.

Tudela, P. (1992). Atención. En J. L. F. Trespalacioes y P. Tudela (Eds), Atención y Percepción. Madrid: Alhambra.

Wechsler, D. (2005). Manual técnico y de interpretación del WISC-IV. Madrid TEA Ediciones S.A.

Yang, P., Cheng, C. P., Chang, C. L., Liu, T. L., Hsu, H. Y., y Yen, C. F. (2013). Wechsler Intelligence Scale for Children 4th edition-Chinese version index scores in Taiwanese children with attention-deficit/hyperactivity disorder. Psychiatry and Clinical Neurosciences, 67(2), 83-91. 
Zhu, J. y Chen, H. (2013). Clinical Utility of Cancellation on the WISC-IV. Journal of Psychoeducational Assessment, 31(6), 527-537.

Recibido: $30-07-2020$

Acceptado: 09-01-2021 